\title{
Relevant, less relevant and irrelevant isokinetic strength test parameters: Some critical comments
}

Zeevi Dvir

Department of Physical Therapy, Sackler Faculty of Medicine, Tel Aviv University, 69978 Israel

Received 3 March 2013 - Accepted 21 March 2013

\begin{abstract}
Isokinetic dynamometry is now widely recognized as the standard tool for assessing muscle performance. However there is still quite a diversity in terms of the report document that is produced following the test/s reflecting various approaches taken by the manufacturers of these instruments. Of particular significance is the use of outcome parameters i.e. those 'numbers' according to which, interpretation regarding muscle performance level may be judged. It appears that some of the parameters that became mainstays of isokinetic reports are redundant at best and misleading, at worst. This short paper is not intended to be a comprehensive review of all outcome parameters but a commentary that sheds light on the main issues concerning this topic.
\end{abstract}

Key words: Isokinetics, outcome measures, parameters, validity

Résumé. Paramètres pertinents, moins pertinents et non pertinents dans les tests isocinétiques : quelques commentaires critiques.

La dynamométrie isocinétique est aujourd'hui largement reconnue comme étant le « gold standard » de l'évaluation de la performance musculaire. Cependant, il existe encore une multitude de rapports que produit chaque dynamomètre lors de tests musculaires traduisant une grande diversité des approches adoptées par les fabricants. Une importance toute particulière est donnée aux paramètres chiffrés puisqu'ils permettent de quantifier des performances musculaires. Il semble cependant que certains de ces paramètres, qui apparaissent dans tous les rapports isocinétiques, soient devenus redondants dans le meilleur des cas et même trompeurs dans le pire des cas. Ce court article n'est pas destiné à être une analyse complète de tous les paramètres isocinétiques, mais plutôt un argumentaire mettant en evidence les principales interrogations relatives à ces derniers.

Mots clés : Isocinétisme, mesures, paramètres, validité

\section{Introduction}

Evaluation of muscular strength is an issue that attracted people's attention since they started appreciating human physical capacity, particularly as such was critically important for one's status and livelihood, let alone life. However measurement of muscle strength, in the strictest meaning of the word, could not start until it became possible to quantify the magnitude of resistance exerted against the tested muscles. The dedicated instrument for this objective, was termed dynamometer and it was therefore developed to record human strength along a continuum, to remove the need for a dead-weight or biological standard, and to measure many different groups of muscles, not just those of lifting or pushing. Thus the first dynamometers were not described before the 18th century; the Graham-Desaguliers dynamometer was developed in
London in 1763 to measure human muscular force, apparently in such a way that synergistic muscles could not impart a false mechanical advantage to the test while the Regnier dynamometer, invented in Paris in 1798, was applied to human muscle force measurement although it was originally intended to measure the traction properties of artillery-horses (Pearn, 1978).

However, these instruments never gained sufficient reputation and no further mention of them, particularly in the wide clinical usage, could be traced. It can therefore safely be stated that the first attempt to systematically assess muscle strength was by Martin and Lovett in 1915 in response to the pressing need to define/describe the extent of muscular weakness that left hundreds of thousands of victims of the poliomyelitis epidemic partially or fully paralyzed (Cuthbert \& Goodheart, 2007). However, the system devised by these physicians - Manual Muscle 
Testing (MMT) - was a qualitative one involving no actual measurement. In fact, it was a test that was partly based on the tester's visual observation or palpation and part on the sensation of his own muscular tension or skin pressure. As a result, MMT was widely criticized suggesting that its clinical utilization be reserved to cases of debilitating weakness only (Sapega, 1990), while other recommended its use to neuromuscular patients (Cuthbert \& Goodheart, 2007).

At any rate, save sporadic attempts to standardize a tool for measuring muscle strength, it was not before 1954 when the first true dynamometer became commercially available - the hand grip dynamometer (HGD) which allowed clinicians to quantify the force exerted during grip at five different flexion positions (rungs) of the hand (Bechtol, 1954). Incorporating elements such as (muscle) length-force relationship, proximal stabilization, bilateral comparison, isometric contractions and endurance, the HGD offer a valid determination of grip strength/weakness based on the position of maximal force output, invariably reached among healthy individuals at rungs 2 or 3 . As a result and to this date, the HGD is the tool of choice among hand clinicians for quantitatively assessing unilateral weakness in grip. Moreover, based on the HGD, the hand pinch strength meter was developed, based in part on the abovementioned principles.

Parallel to the HGD, the use of isometric devices for muscle strength measurement continued to be widely advocated by rehabilitation and sports medicine clinicians as well as by human performance experts. However, the effort to develop such a tool for general isometric muscle strength measurement did not succeed for many years until the first hand-held digital dynamometer (HHD) became commercially available. Besides being impaired with some significant limitations, by the time it was on the market (beginning of the 1990s), the absolutely more encompassing and technologically advanced (albeit on a totally different price scale) isokinetic dynamometer (ISD) already enjoyed the wide recognition of the medical, research and applied exercise science communities.

The isokinetic principle was scientifically introduced by Hilsop and Perrine (1967). The instrument described (Cybex) was a purely passive system capable of measuring either concentric or isometric exertions. Its accommodating resistance feature was derived from an hydraulic piston which allowed rotation of the lever arm at six prescribed velocities $(30-180 \%$ s, at a $30 \%$ interval) based on the adjustable diameter of the oil outlet valve. The trace of the moment produced by the tested muscles: invariably the quadriceps and the hamstring, was registered on paper. This trace allowed testers to visually identify the point on the curve which coincided with the maximal moment output or the maximal strength of the tested muscle. Using a simple ruler and conversion factor, the $\mathrm{Nm}$ value of this point, was calculated and termed quite erroneously as the peak torque (PT) although from the mechanical viewpoint, peak moment is the correct term. Applying a range of velocities and based on the individual velocity related-PTs, the concentric branch of the muscle torque-velocity curve could be obtained. In addition to the individual muscles PT, the antagonistic strength relationships, typically the ratio: $\mathrm{PT}(\mathrm{Hams}) / \mathrm{PT}(\mathrm{Quad})$, could be calculated with respect to the test angular velocity.

However, with the increasing incorporation of computers into measurement systems, the original data recording system of the ISD was upgraded to include an A-to-D converter while the torque curve was now displayed on the computer screen. With the facility offered by the computer for instant processing and displaying of test data, the manufacturers were presented with an opportunity to produce a report sheet. The latter consisted of the graphic presentation of the tested torque curves and an ever growing number of test parameters, heretofore outcome measures, which included the average torque, total work, maximal power, angle of and time to peak torque, torque acceleration energy etc; as well as reference to the $\mathrm{H} / \mathrm{Q}$ ratios. Whether or not these parameters carried any scientific or, especially, clinical significance was never disclosed by the manufacturers while users kept referring to them and including some or all in scientific manuscripts. The main objective of this paper is therefore to offer some insight regarding the utility of several of these outcome parameters.

Without much elaborating, it should be mentioned that in order for an outcome parameter to be clinically efficient it has to fulfill two main requirements namely it has to be reproducible and it has to be valid. In recent years the responsiveness of a parameter, i.e. its sensitivity to change, has been mentioned as an independent factor. One can also add the ability of a given parameter to distinguish between distinct groups, typically healthy individuals vs. patients expressed as the specificity and sensitivity of the parameter. While numerous studies looked into the requirement of reproducibility, the issue of validity of isokinetic outcome parameters has been accorded lesser attention. This may be explained by the fact that isokinetic dynamometry is widely considered the gold standard in muscle testing. Since validity very often involves comparison to other systems, using inferior techniques to validate isokinetics is almost contradiction in terms. On the other hand, studies regarding responsiveness, specificity and sensitivity are still lacking.

\section{Peak torque, average torque and peak force}

Figure 1 depicts a typical torque-angular position curve. The peak value of the curve is the PT. It should be remembered that the torque values may be derived directly from a torque sensor that is co-aligned with the axis of the motor or from multiplication of the force by the length of the lever arm. In the latter case, the force is obtained from a force sensor that is embedded in the distal attachment 


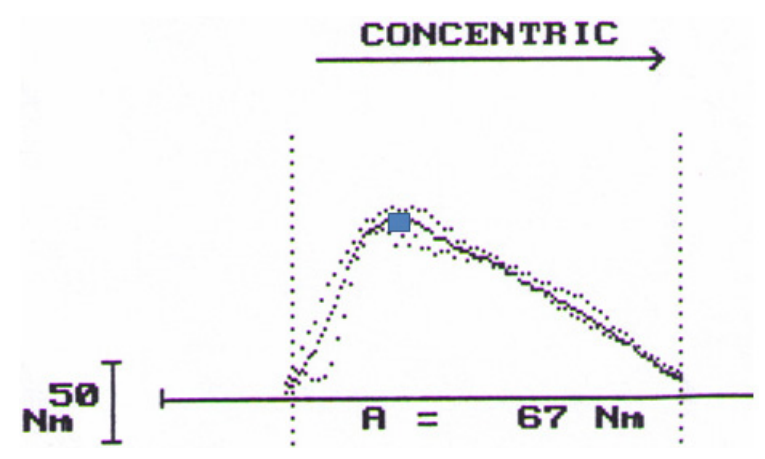

Fig. 1. The peak torque (PT). The value of $67 \mathrm{Nm}$ is the average torque between the vertical dashed lines. The PT, based on the system's software is $106 \mathrm{Nm}$.

that slides on the lever arm, while the effective length of the lever arm is fixed. Thus the force rather than the torque is the basic physical entity or outcome measure since the latter is obtained by multiplying the force by a constant. The significance of this distinction which is of fundamental importance follows below.

Since the inception of isokinetic dynamometery, the PT has been the quintessential outcome parameter serving in this role in thousands of publications. Its validity was vindicated time and again as the measure for the maximal mechanical potential of the muscle. The four main issues addressed by the PT are:

1) To characterize the strength of specific muscle groups in distinct cohorts: apparently healthy children, adolescents, adults and elderly people, males and females; in individuals suffering from specific pathologies or disorders and in people participating in various sports: amateurs as well as professionals.

2) When appropriate, to use reference values collected in (1) for screening, particularly in various sports (Dervišević \& Hadžić, 2012).

3) To assess unilateral weakness of a given muscle/muscle group due to injury or disease, based on the strength of its contra-lateral counterpart

4) Where the disorder is of a systemic nature, to assess the weakness based on well established reference values

5) To assess muscular imbalance using the simple concentric vs. concentric $\mathrm{PT}$ ratio e.g. the shoulder $\mathrm{PT}_{\text {internal rotation }} / \mathrm{PT}_{\text {external rotation }}$ or the dynamic control ratio (DCR) (Dvir, et al. 1989), which relates to the eccentric vs. concentric $\mathrm{PT}$ ratio e.g. $\mathrm{PT}$ Hams-eccentric $/ \mathrm{PT}_{\text {quadriceps concentric }}$ (Hecc/Qcon).

In a recent comprehensive review, the reproducibility of the PT, predominantly with respect to the abovementioned first issue, and in terms of all commonly measured muscle groups has been supported with the singling out of high test velocities as having a compromising effect (Caruso, Brown, \& Tufano, 2012).

The PT remains stable also when using the short rather than the 'standard' range of motion as shown

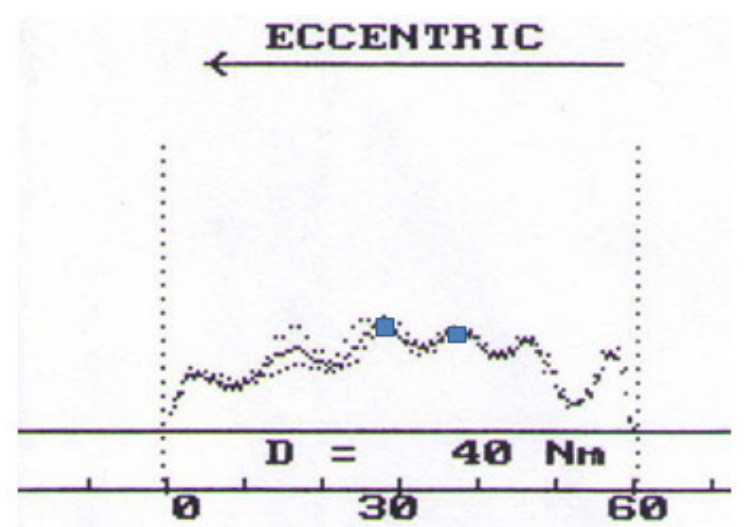

Fig. 2. An isokinetic torque curve characterized by multiple peaks. The AvT works as an "integrator".

with respect to knee (Reichard, Croisier, Katz-Leurer, Malnati, \& Dvir, 2005) and wrist (Reichard, Katz-Leurer, Rubinstein, Croisier, \& Dvir, 2010) flexors and extensors under both concentric and eccentric conditions. In other words, the PT effectively reflects the combination of the tension (concentric or eccentric) and the lever arm under a specific test protocol. Interestingly, the relationship between static and dynamic PT are not perfectly correlated although the lower the velocity, the higher is the correlation (Dvir, Steinfeld-Cohen, \& Peretz, 2002).

Whereas the normal torque-angular position curve is a fairly smooth one with a distinct maximum, enabling an unequivocal determination of the $\mathrm{PT}$, such is not the case when the torque signal oscillates (Fig. 2). With multiple peaks, deciding which peak is associated with the true maximum may be erroneous and hence of lesser or no clinical relevance. In such cases, where the irregular curve is associated with the involved side and when the objective is quantifying this side's weakness, a possible solution is to use the average torque (AvT) as the outcome measure. The AvT may relate to the full test RoM or part of it and its main role is to 'clean' the signal and enable a valid comparison to the uninvolved side within the prescribed sector. In a paper which looked into the PT-AvT interactions, it was indicated that as expected, these outcome measures were strongly correlated and hence could be applied interchangeably when conducting unilateral weakness analysis (Dvir \& David, 1995). However, for most of the objectives above, the AvT is of no relevance as it does not define the maximal potential of the muscle.

The PT may be less than ideal in other instances as well. It should be remembered that the alignment of the motor axis with the joint axis is a critical step in the test protocol. However, against a fixed mechanical axis, biological joints are distinguished by being polycentric, namely the axis is instantaneous and is defined in three dimensions. Therefore the alignment process is in fact one of optimization, attempting to reduce the error to a minimum by finding a "mean" common axis which passes through an easy to find an anatomical landmark e.g. the 
head of the fibula or the lateral femoral epicondyle in the case of the knee. While for relatively simple joints like the elbow, the "mean" axis is less of a problem, in more complex joint systems like the spine, alignment is a major issue reflected by the controversy regarding the optimal location of this 'mean' axis: the greater trochanter, at the level of L5-S1 or L2-L3 or otherwise (Dvir, 2004). Using these different 'axes' inevitably leads to non-uniformity in the spine test protocols, which ultimately results in significantly different test findings. Consequently, expressing spine muscles strength in moment-based Nm units may render the findings less relevant or in extreme cases, even irrelevant.

On the other hand, if in the case of the spine extensors, instead of using the PT, the force exerted on the force pad is used as the outcome measure - now PF in $\mathrm{N}$ - the stability of the measurement is significantly increased. Such a Newton-based expression is also functionally valid in terms of the maximal ability to push backward (extend the spine) while reducing the error of measurement (Rantanen, Airaksinen, \& Pentinnen, 1994). Indeed when relating to force rather than to moment, the center of joint rotation becomes irrelevant as long as the motion spans a short arc and the measured quantity is force. This was clearly shown in a study of trunk extension strength using the principle of short range of motion where the reproducibility of the $\mathrm{PF}$ was strongly supported (Dvir \& Keating, 2001).

Another example is that of testing isokinetically grip strength (Dvir, 1997). This function is normally measured using an isometric device. However, some ISDs offer a dedicated grip device where the outcome measure $(\mathrm{PT})$ is expressed in Nm units. But relative to which axis? Given the large number of involved joints, from the wrist and distally toward the finger joints, using a moment-based value becomes an irrelevant representation. If however, the force exerted by the fingers is the outcome parameter, the measurement unit becomes clinically valid. Thus the peak force $(\mathrm{PF})$ and not the $\mathrm{PT}$ is the relevant isokinetic outcome measure despite the fact that the typical ISD functions as an angular actuator. In this context it is worth mentioning that linear isokinetic dynamometers which are mainly used for legpress testing and training, should refer to force or $\mathrm{PF}$ rather than the torque although in principle, it is possible to derive the 'net moment' acting on the knee if other kinematic parameters are known. Thus as a deviation from the main isokinetic outcome parameter (the PT), the equally fundamental outcome parameter - the PF - is a clinically relevant outcome measure.

\section{Angle of PT and Time to PT}

The force or torque signal has some other characteristics, in addition to their peak value. Among these the time to peak torque (TPT, in s) and particularly the angle of peak torque (APT, in ${ }^{\circ}$ ) became the central issue of quite a number of publications. It was alleged that the TPT related basically to the explosive nature of the dynamic contraction, similar to the role the rate of force development (RFD) fulfills with respect to powerful isometric efforts. On the other hand the APT ostensibly reflects some structural properties of the muscle which may lead to a delay in reaching the PT. In principle a correlation between these two parameters is expected namely a shorter TPT should be expressed also in a smaller angle. However, as mentioned in the Introduction, a sine qua non condition for the clinical relevance of an outcome measure is its acceptable reproducibility and studies focusing on the APT and TPT in this context have largely been not encouraging. Most studies date back to the 1990's but recently this topic has been systematically studied with respect to the bilateral concentric strength of the knee flexors and extensors at 60 and $180 \%$ s (Bernard, et al., 2011). The "test" and "retest" sessions were performed within the same day, $45 \mathrm{~min}$ apart. This paradigm is particularly favorable for a high reproducibility while the cohort of 29 well trained athletes was another positive factor. In spite of this set-up, both the APT and TPT demonstrated moderate to good ICCs: $0.5-0.8$ and $0.6-0.8$ respectively. With other studies reporting conflicting results, the authors have rightfully concluded that given their low reproducibility, neither of these outcome parameters should be used for the characterization of muscular adaptations in athletes. Worth mentioning, a recent unpublished study comparing the concentric APT of the quadriceps and hamstring in soccer players who underwent reconstruction of the ACL with players whose ACL was apparently intact (Cordeiro, Cortes, \& Pezarat-Correia, 2013). It was found that only in a single instance: quadriceps at $300 \% \mathrm{~s}$ a significant difference existed between the groups with a delayed PT in the former. Significantly, no such difference was indicated at 60 and $180 \%$ s, and it was totally absent in the hamstring. This ostensibly could indicate that the APT could serve as an outcome measure. However, at a velocity of $300 \%$ s, the contraction is no longer of an isokinetic nature. Furthermore, no reproducibility study was undertaken and therefore the significance of the finding requires further research. Consequently, at the present moment, it seems safe to state that while the APT is of a lesser relevance at best, the TPT is of no clinical relevance.

\section{Torque acceleration energy}

This parameter was suggested as an outcome measure and was part of the report sheet of the Cybex dynamometer. It related to the total work exerted during the first $1 / 8$ of a second of a concentric contraction and allegedly reflected the explosive nature of the muscle (Davies, 1992). However, a reproducibility analysis of this parameter revealed test-test correlations of negligible magnitude and hence the torque acceleration energy is an irrelevant isokinetic outcome measure (Madsen, 1996). 


\section{Rate of force (torque) development}

The rate of force (torque) development (RFD, or RTD) is the maximal slope of the rising branch of the force (torque) curve. It is usually performed using isometric devices. Since the lever arm of isokinetic dynamometers can be locked, RTD may be derived. However, trying to calculate the RTD under dynamic circumstances introduces a further interaction i.e. between the motor response and the recruitment process. The higher the pre-set velocity, the longer it takes the lever arm (motor) to reach it. In the transitional phase, the resistance corresponds to the inertia of the lever and the body segment but is not the maximally accommodating one. This may cause flutter in the torque curve and eventually limit even further the reproducibility of this parameter. Thus it does seem that present knowledge precludes the use of dynamic RTD as a relevant outcome parameter.

\section{Work and power}

Work and power are two closely related parameters. Per definition work (W), which is expressed in Joules, is the integral of the force by distance: $W=\int \mathrm{F} d \mathrm{x}$ where $\mathrm{dx}$ refers to the infinitesimal change in the linear displacement. This definition serves simply and efficiently linear trajectories. For example this equation applies for calculating the work done by the quadriceps (predominantly) when overcoming the resistance in a maximal isokinetic leg-press pattern. Since the force varies along the linear trajectory, every small change in the displacement (dx) must be multiplied by the corresponding force and added piecewise to determine the final joule value of the work. When work is being done in an angular fashion the equation takes the following form, $W=\int \mathrm{T} d \varphi$, where $d \varphi$ refers to the infinitesimal change in the angular displacement ad $\mathrm{T}$ is the torque. The unit for angular work is equally the joule $(\mathrm{J})$. Angular work is a particularly suitable outcome parameter for standard isokinetic dynamometers since all are of the angular type. Figure 3 depicts angular work as the area under the isokinetic curve. It should be noted that unlike the PT or PF which are well defined single points (assuming curve regularity), Work, and inevitably Power, relate to the relevant end points, a direct consequence of the mathematical definition of the integral. For example, suppose in testing of the shoulder abductors over a RoM of $60^{\circ}$ the PT is $66 \mathrm{Nm}$. For this particular RoM the work done by the abductors is equal to:

$$
W=\int_{0}^{60} T \mathrm{~d} x
$$

If on the other hand, the RoM is narrowed, e.g. to $30^{\circ}$ in such way that it still contains the point of $\mathrm{PT}$, while the test velocity is reduced proportionately, the PT will likely remain very similar (as previously discussed with respect

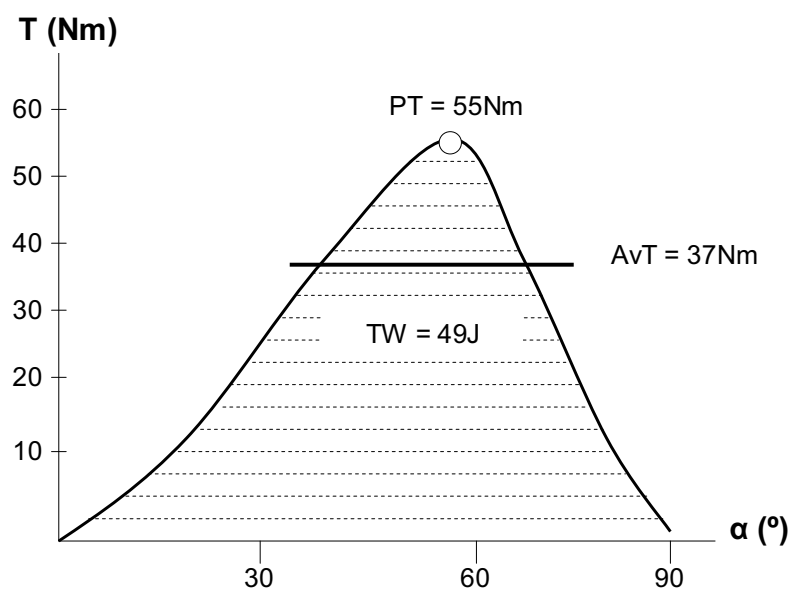

Fig. 3. Total work, PT and AvT demonstrated on a simulated isokinetic curve. $X$ axis - the angular position of the joint; $Y$ axis (T) - torque.

to short RoM testing) while Work will be reduced to reflect the fact that the limits of integration are now 0 to $30^{\circ}$. Thus against a relative invariance of the PT (or PF), Work depends on the RoM, limiting its generalizability.

Power is defined as the rate at which work is being done over time $P=W / t$. If the instantaneous power is sought, this equation takes the differential form of: $P=\mathrm{d} W / \mathrm{d} t$. In terms of isokinetic analysis, the preferred form is that of average power (heretofore, Power) which is equal to the total contractional work divided by the contraction duration. Power is expressed in watts. For example if the total work/contraction is $120 \mathrm{~J}$ and the time to complete the contraction is $0.8 \mathrm{~s}$ then the average power $(\mathrm{P})$ is $120 / 0.8-150 \mathrm{~W}$. In much the same way like work, Power is may be defined between endpoints; maximal power $\left(\mathrm{P}_{\max }\right)$ could correspond to that sector in the torque curve in which the work reaches its maximum value. As the denominator in the power equation is given in fixed time units which with current sampling rates could reach $5 \mathrm{~ms}$, Work and Power are absolutely correlated.

It should now be noticed that in highly regular isokinetic curves there is a very strong correlation, or using the mathematical expression "linear dependence", between the PT and the integral under the curve - the Work. This correlation stems mainly from the fact that the PT is a major factor within the integration process. As the curve becomes increasingly irregular (e.g. with more oscillations) this strong relationship diminishes. However, assuming good regularity Work may then be a redundant outcome parameter since the PT conveys whatever information is needed to characterize the strength of the muscle. It is thus surprising that users of isokinetics often insist on quoting the work done by the tested muscle, alongside reference to the PT. As it is the torque magnitude that dictates eventually the amount of work (assuming standard range of motion) the PT is the fundamental and clinically relevant outcome parameter, not Work (but see 
below for the role of work in fatigue studies). Taking this issue one step further into the Power domain, this approach is equally vindicated. As mentioned before, since isokinetic Power is the result of dividing Work by a fixed time duration, there is a 1:1 relationship between Work and Power within the same isokinetic range of motion. Indeed a study of healthy subjects and patients undergoing long-term steroid therapy for rheumatic diseases these relationships were examined based on testing of quadriceps at 30,60, 90, and $120 \%$ s. (Rothstein, Delitto, Sinacore, \& Rose, 1983). At all velocities and for subjects and patients alike, high correlations were found between PT and P. Population- and velocity-specific linear regression equations were calculated that allowed for predictions of $\mathrm{P}$ from the more easily obtained clinical measure of PT. Doubtless, had the same analysis been extended to include the isokinetic Work, the very same correlations would have been indicated. It should also been mentioned that failing to reach even higher correlations could be attributed to intrinsic (discounting joint poly-centricity) as well to extrinsic factors (e.g. system accuracy, velocity control). Consequently as far as strength testing of muscles is concerned, the parallel use of Work and Power is unjustified, creating a redundancy and over-burdening the user with information that has no further importance.

On the other hand, Work may be an important outcome parameter in maximal effort fatigue analysis. Although outside the confines of this paper, which deals with strength related parameters, some reference is deserving. In the mechanical context, muscle fatigue analysis generally looks into the decease in the muscle's mechanical output. This may be reflected in a decrease in the maximal torque or in Work. As both are closely correlated, one is redundant. However, some studies have focused on Work as the preferred outcome parameters and among them the comprehensiveness of assessing reproducibility was especially compelling (Saenz, et al., 2010). In this multi-center test-retest study, a concentric fatigue protocol of the knee flexors and extensors consisting of 40 maximal repetitions was carried out at $120 \%$ s. The main findings indicated that the total work throughout the effort as well as the work done during the first and last third of the series were highly reliable. Since Work is the integral of the torque curve, it may represent in a better way the total energy expenditure and therefore should serve as a relevant outcome measure. Also, in this particular instance, work is taken along the full range of motion and is therefore easier to interpret.

Likewise, the use of Power as an independent outcome measure has been advocated with respect to various sporting activities. The logic behind this approach was that sports require fast recruitment and high PTs. Due to the previous, testing at high speed was deemed more valid while the outcome parameter of Power could better reflect specific skills. However, the distinguishing power of Power in terms of showing a higher differential power (sensitivity + specificity) than e.g. the $\mathrm{PT}$ regarding specific sports, may have never been proven. Such a study should have also assessed the comparative reproducibility of both outcome parameters and although speculative, it is hypothesized that that of the PT, would have prevailed. It should also be mentioned that high speed $(>180 \%$ s) testing was shown to result in sizeable, if any, non-isokinetic sectors and therefore the validity of calling defining such tests as isokinetic is much in question. Thus, tests carried out at $240,300 \%$ and even beyond are basically ballistic with all associated implications.

\section{Conclusions}

This short commentary highlights a few fundamental issues relating to the use of various outcome measures in isokinetics. It is suggested that the well established relevant outcome parameters are the peak torque $(\mathrm{PT})$ and peak force $(\mathrm{PF})$. Work $(\mathrm{W})$ and power $(\mathrm{P})$, by virtue of being highly correlated with $\mathrm{PT}$ are of much lesser relevance in strength testing. Nevertheless, W (along the $\mathrm{PT}$ ) seems to be a relevant outcome parameter in fatigue testing. Other isokinetically-derived outcome parameters such as rate of force development (RFD), torque acceleration energy (TAE), angle of peak torque (APT) and time to peak torque (TPT), seem largely irrelevant first and foremost for lack of clinically established reproducibility. Among these the APT, deserves further research.

\section{Bibliography}

Bernard, P.L., Amato M., Degache F., Edouard P., Ramdani S., Blain H., Calmels P., \& Codine P. (2012). Reliability of the time to peak torque and the joint angle at peak torque of the isokinetic dynamometer. Annals of Physical and Rehabilitation Medecine, 55, 241-251.

Bechtol, C.O. (1954). Grip test: Use of dynamometer with adjustable handle spacings. Journal of Bone and Joint Surgery, 36, 820-824.

Caruso, J.F., Brown, L.E., \& Tufano, J.J. (2012). The reproducibility of isokinetic dynamometry data. Isokinetics and Exercice Science, 20, 239-255.

Cordeiro, N., Cortes, N., \& Pezarat-Correia, P. (2013). Specific isokinetic angle peak torque of fast angular knee velocities on soccer players after ACL reconstruction. Submitted.

Cuthbertn S.C., \& Goodherat, G.J. (2007). On the reliability and validity of manual muscle testing: a literature review. Chiropractic \& Osteopathy, 15, 4-20.

Davies, G.E. (1992). A Compendium of Isokinetics in clinical Usage. 4th ed. Onalaska, WIS: S \& S Publishers.

Dervišević, E., \& Hadžić, V. (2012). Quadriceps and hamstrings strength in team sports: Basket ball, fooball and volleyball. Isokinetics and Exercice Science, 20, 293-300.

Dvir Z. (1997). The measurement of dynamic finger flexion strength using isokinetic dynamometry. Clinical Biomechanics, 12, 473-481. 
Dvir, Z. (2004). Isokinetics: Muscle testing, interpretation and clinical applications. 2nd ed. Edinburgh: ElsevierChurchill Livingstone.

Dvir, Z., \& David, G. (1995). Average or peak moment: which is the more suitable parameter to represent isokinetic muscle strength? Isokinetics and Exercice Science, 5, 93-97.

Dvir, Z., \& Keating, J. (2001). The reproducibility of isokinetic trunk extension: A study using very short range of motion. Clinical Biomechanics, 16, 627-630.

Dvir, Z., Eger, G., Halperin, N., \& Shklar, A. (1989). Thigh muscles activity and ACL insufficiency. Clinical Biomechanics, 4, 87-91.

Dvir, Z., Steinfeld-Cohen, Y., \& Peretz, C. (2002). The identification of feigned isokinetic shoulder flexion weakness in normal subjects. American Journal of Physical Medecine \& Rehabilitation, 81, 178-183.

Hilsop, H.J., \& Perrine, J.J. (1967). The isokinetic concept of exercise. Physical Therapy, 4\%, 114-117.

Madsen, O.R. (1996). Torque, total work, power, torque acceleration energy and acceleration time assessed on a dynamometer: reliability of knee and elbow extensor and flexor strength measurement. European Journal of Applied Physiology, 74, 206-210.

Pearn, J. (1978). Two early dynamometers. An historical account of the earliest measurements to study human muscular strength. Journal of Neurological Science, 37, 127-134.

Rantanen, P., Airaksinen, O., \& Pentinnen, E. (1994). Paradoxical variations of strength determinants with different rotation axes in trunk flexion and extension strength tests. European Journal of Applied Physiology, $68,322-326$.

Reichard, L.B., Croisier, J.L., Katz-Leurer, M., Malnati, M., \& Dvir, Z. (2005). Testing knee extension and flexion strength at different ranges of motion: an isokinetic and electromyographic study. European Journal of Applied Physiology, 95, 371-376.

Reichard, B., Katz-Leurer, M., Rubinstein, M., Croisier, J.L., \& Dvir Z. (2010). Short range of motion isokinetic testing of wrist flexors and extensors strength in normal subjects and patients with carpal tunnel syndrome. Journal of Strength and Conditioning Research, 24, 1866-1873.

Rothstein, J.M., Delitto, A., Sinacore, D.R., \& Rose, S.J. (1983). Electromyographic, peak torque, and power relationships during isokinetic movement. Physical Therapy, 63, 926-933.

Saenz, M., Avellanet, M., Hijos, J., Chaler, J., Garreta, R., Pujol, E., Sandoval, B., Buen, C., \& Farreny, A. (2010). Knee isokinetic test-retest: a multicenter $\mathrm{knw}$ isokinetic test-retest study of a fatigue protocol. European Journal of Physical Medecine and Rehabilitation, 46, 81-87.

Sapega, A.A. (1990). Muscle performance evaluation in orthopedic practice. Journal of Bone \& Joint Surgery, 72, 1562-1574.

Webber, S.C., \& Porter, M.M. (2010). Reliability of ankle isometric, isotonic, and isokinetic strength and power testing in older women. Physical Therapy, 90, 1165-1175. 